Document downloaded from:

http://hdl.handle.net/10251/71246

This paper must be cited as:

Bonet Solves, JA. (2015). The spectrum of Volterra operators on weighted spaces of entire functions. Quarterly Journal of Mathematics. 66:799-807. doi:10.1093/qmath/hav019.

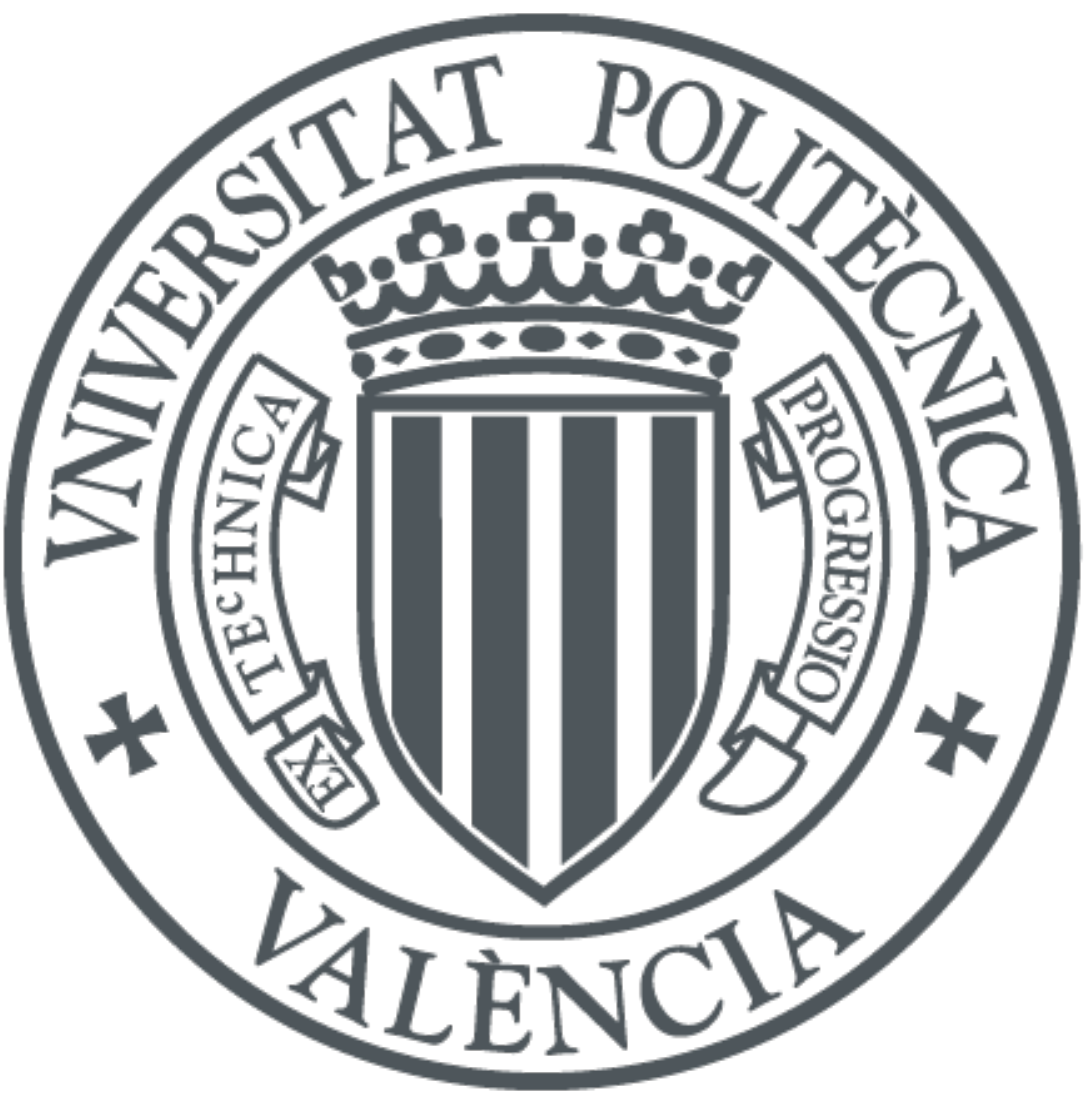

The final publication is available at

http://dx.doi.org/doi:10.1093/qmath/hav019

Copyright Oxford University Press (OUP)

Additional Information 


\title{
The spectrum of Volterra operators on weighted spaces of entire functions
}

\author{
José Bonet
}

\begin{abstract}
We investigate the spectrum of the Volterra operator $V_{g}$ with symbol an entire function $g$, when it acts on weighted Banach spaces $H_{v}^{\infty}(\mathbb{C})$ of entire functions with sup-norms and when it acts on Hörmander algebras $A_{p}$ or $A_{p}^{0}$.
\end{abstract}

\section{Introduction, notation and preliminaries}

The aim of this note is to investigate the spectrum of the Volterra operator when it acts continuously on a weighted Banach space of entire functions $H_{v}^{\infty}(\mathbb{C})$. Our main result is Theorem 2.3. The characterizations of continuous Volterra operators on $H_{v}^{\infty}(\mathbb{C})$ obtained by Taskinen and the author in [13] play an important role. The present research originated with a question of A. Aleman in a meeting celebrated in Granada in February 2015 in which the results in [13] were presented. In Section 3 we study the spectrum of Volterra operators acting on a (DFN) Hörmander algebra $A_{p}$ in Theorem 3.4, and on a Fréchet Hörmander algebra $A_{p}^{0}$ in Theorem 3.5.

In what follows $H(\mathbb{C})$ denotes the space of entire functions. The space $H(\mathbb{C})$ is a Fréchet space endowed with the compact open topology. The differentiation operator $D f(z)=f^{\prime}(z)$, the integration operator $J f(z)=\int_{0}^{z} f(\zeta) d \zeta$ and the multiplication operator $M_{h}(f)=h f, h \in H(\mathbb{C})$, are continuous on $H(\mathbb{C})$.

Given a non-constant entire function $g \in H(\mathbb{C})$ with $g(0)=0$, the Volterra operator $V_{g}$ with symbol $g$ is defined on $H(\mathbb{C})$ by

$$
V_{g}(f)(z):=\int_{0}^{z} f(\zeta) g^{\prime}(\zeta) d \zeta \quad(z \in \mathbb{C}) .
$$

For $g(z)=z$ this reduces to the integration operator. Clearly $V_{g}$ defines a continuous operator on $H(\mathbb{C})$. The Volterra operator for holomorphic functions on the unit disc was introduced by Pommerenke [28] and he proved that $V_{g}$ is bounded on the Hardy space $H^{2}$, if and only if $g \in B M O A$. Aleman and Siskakis [3] extended this result for $H^{p}, 1 \leq p<\infty$, and they considered later in [4] the case of weighted Bergman spaces; see also [26]. We refer the reader to the memoir by Peláez and Rättyä [27] and the references therein. Volterra operators on weighted Banach spaces of holomorphic functions on the disc of type $H^{\infty}$ have been investigated recently in [5]. Constantin started in [15] the study of the Volterra operator on spaces of entire functions. She characterized the continuity of $V_{g}$ on the classical Fock spaces and investigated its spectrum. Constantin and Peláez

2010 Mathematics Subject Classification. Primary: 47G10, secondary: 30D15, 30D20, 46E15, 47B07, 47B37, 47B38.

Key words and phrases. Spectrum; integral operator; Volterra operator; entire functions; growth conditions; weighted Banach spaces of entire functions; Hörmander algebras 
[16] characterize the entire functions $g \in H(\mathbb{C})$ such that $V_{g}$ is bounded or compact on a large class of Fock spaces induced by smooth radial weights. See also [13]. Aleman and Constantin [1] and Aleman and Peláez [2] investigate the spectra of Volterra operators on several spaces of of holomorphic functions on the disc.

A weight $v$ is a continuous function $v:[0, \infty[\rightarrow] 0, \infty[$, which is non-increasing on $[0, \infty[$ and satisfies $\lim _{r \rightarrow \infty} r^{m} v(r)=0$ for each $m \in \mathbb{N}$. We extend $v$ to $\mathbb{C}$ by $v(z):=v(|z|)$. For such a weight, the weighted Banach space of entire functions is defined by

$$
H_{v}^{\infty}(\mathbb{C}):=\left\{f \in H(\mathbb{C})\left|\|f\|_{v}:=\sup _{z \in \mathbb{C}} v(|z|)\right| f(z) \mid<\infty\right\},
$$

and it is endowed with the weighted sup norm $\|\cdot\|_{v}$. Changing the value of $v$ on a compact interval does not change the space and gives an equivalent norm. Spaces of this type appear in the study of growth conditions of analytic functions and have been investigated in various articles, see e.g. $[10,11,22]$ and the references therein.

For an entire function $f \in H(\mathbb{C})$, we denote $M(f, r):=\max \{|f(z)||| z \mid=r\}$. Using the notation $O$ and $o$ of Landau, $f \in H_{v}^{\infty}(\mathbb{C})$ if and only if $M(f, r)=O(1 / v(r)), r \rightarrow \infty$. By $C, C^{\prime}, c$ etc. we denote positive constants, the value of which may vary from place to place.

Let $T: X \rightarrow X$ be a continuous operator on a space $X$. The resolvent of $T$ on $X$ is the set $\rho(T, X)$ of all $\lambda \in \mathbb{C}$ such that $T-\lambda I: X \rightarrow X$ is bijective and has a continuous inverse. Here $I$ stands for the identity operator on $X$. The spectrum $\sigma(T, X)$ of $T$ is the complement in $\mathbb{C}$ of the resolvent. The point spectrum is the set $\sigma_{p t}(T, X)$ of those $\lambda \in \mathbb{C}$ such that $T-\lambda I$ is not injective. Observe that in this paper we consider operators defined not only on Banach spaces, but also on more general spaces: $H(\mathbb{C})$ and the Hörmander algebra $A_{p}^{0}$ are Fréchet spaces and the Hörmander algebra $A_{p}$ is the dual of a Fréchet space. Accordingly, when we refer to a space, we mean a Hausdorff locally convex space. We refer the reader to [25] for results and terminology about functional analysis.

At this point we include some preliminary results, in particular about the spectrum of the Volterra operator on $H(\mathbb{C})$ that are inspired by [1] and [2]. Let $g \in H(\mathbb{C})$ be a non-constant entire function such that $g(0)=0$ and let $V_{g} f(z)=\int_{0}^{z} f(\zeta) g^{\prime}(\zeta) d \zeta, z \in \mathbb{C}$, denote the Volterra operator associated with $g$, that acts continuously on $H(\mathbb{C})$.

Proposition 1.1 The operator $V_{g}-\lambda I: H(\mathbb{C}) \rightarrow H(\mathbb{C})$ is injective for each $\lambda \in \mathbb{C}$. In particular $\sigma_{p t}\left(V_{g}, H(\mathbb{C})\right)=\emptyset$. Moreover, $0 \in \sigma\left(V_{g}, H(\mathbb{C})\right)$.

Proof. If $\left(V_{g}-\lambda I\right) f=0$, then $\lambda f(0)=0$ and $f(z) g^{\prime}(z)=\lambda f^{\prime}(z), z \in \mathbb{C}$,. If $\lambda=0$, we get $f=0$, since $g$ is not constant. In case $\lambda \neq 0$, we get $f(z)=C \exp (g(z) / \lambda)$, which implies $f=0$, since $f(0)=0$.

The operator $V_{g}$ is not surjective on $H(\mathbb{C})$ because $V_{g} f(0)=0$ for each $f \in H(\mathbb{C})$. Thus $0 \in \sigma\left(V_{g}, H(\mathbb{C})\right)$.

Lemma 1.2 Given $\lambda \in \mathbb{C}, \lambda \neq 0$, and $h \in H(\mathbb{C})$, the equation $f-(1 / \lambda) V_{g} f=h$ has a unique solution given by

$$
f(z)=R_{\lambda, g} h(z)=h(0) e^{\frac{g(z)}{\lambda}}+e^{\frac{g(z)}{\lambda}} \int_{0}^{z} e^{-\frac{g(\zeta)}{\lambda}} h^{\prime}(\zeta) d \zeta, \quad z \in \mathbb{C} .
$$

Proof. This is well known; see e.g. [1, p. 200] or [2, p. 2]. The uniqueness follows from Proposition 1.1. It is enough to substitute in the equation to check the result. Alternatively, the equation implies $f(0)=h(0)$ and that $f$ is the solution of the equation $y^{\prime}-\left(g^{\prime}(z) / \lambda\right) y=h^{\prime}(z)$. The result is also obtained solving this linear equation. 
Proposition 1.3 Let $g \in H(\mathbb{C})$ be a non-constant entire function such that $g(0)=0$. The Volterra operator $V_{g}$ satisfies $\sigma\left(V_{g}, H(\mathbb{C})\right)=\{0\}$ and $\sigma_{p t}\left(V_{g}, H(\mathbb{C})\right)=\emptyset$.

Proof. This is a direct consequence of Proposition 1.1 and the continuity of the operator $R_{\lambda, g}: H(\mathbb{C}) \rightarrow H(\mathbb{C}), \lambda \neq 0$.

Lemma 1.4 Let $X \subset H(\mathbb{C})$ be a locally convex space that contains the constants and such that the inclusion $X \subset H(\mathbb{C})$ is continuous. Assume that $V_{g}: X \rightarrow X$ is continuous for some non-constant entire function $g$ such that $g(0)=0$. Then

$$
\{0\} \cup\left\{\lambda \in \mathbb{C} \backslash\{0\} \mid e^{\frac{g}{\lambda}} \notin X\right\} \subset \sigma\left(V_{g}, X\right) .
$$

If $X$ is a Banach space, then

$$
\{0\} \cup \overline{\left\{\lambda \in \mathbb{C} \backslash\{0\} \mid e^{\frac{g}{\lambda}} \notin X\right\}} \subset \sigma\left(V_{g}, X\right) .
$$

Proof. Since $X$ contains the constants, $V_{g}$ is not surjective and $0 \in \sigma\left(V_{g}, X\right)$. If $\lambda \notin$ $\sigma\left(V_{g}, X\right)$, then $\lambda \neq 0$ and the operator $R_{\lambda, g}: X \rightarrow X$ defined in Lemma 1.2 is continuous. In particular, $R_{\lambda, g}(1)=e^{\frac{g}{\lambda}} \in X$. This implies the desired inclusion. Recall that in case $X$ is a Banach space, $\sigma\left(V_{g}, X\right)$ is compact.

Lemma 1.5 Let $X \subset H(\mathbb{C})$ be a locally convex space that contains the constants and such that the inclusion $X \rightarrow H(\mathbb{C})$ is continuous. Assume that $V_{g}: X \rightarrow X$ is continuous for some non-constant entire function $g$ such that $g(0)=0$. The following conditions are equivalent:

(i) $\lambda \in \rho\left(V_{g}, X\right)$.

(ii) $R_{\lambda, g}: X \rightarrow X$ is continuous.

(iii) (a) $e^{\frac{g}{\lambda}} \in X$, and

(b) $S_{\lambda, g}: X_{0} \rightarrow X_{0}, S_{\lambda, g} h(z):=e^{\frac{g(z)}{\lambda}} \int_{0}^{z} h^{\prime}(\zeta) e^{-\frac{g(\zeta)}{\lambda}} d \zeta, z \in \mathbb{C}$, is continuous on the subspace $X_{0}$ of $X$ of all the functions $h \in X$ with $h(0)=0$.

Proof. This follows directly from the definitions.

Lemma 1.6 Let $X \subset H(\mathbb{C})$ be a locally convex space that contains the constants and such that the inclusion $X \rightarrow H(\mathbb{C})$ is continuous. Let $X_{0}$ be the subspace of $X$ of all the functions $h \in X$ with $h(0)=0$. The following conditions are equivalent for $\lambda \in \mathbb{C} \backslash\{0\}$.

(i) $S_{\lambda, g}: X_{0} \rightarrow X_{0}, S_{\lambda, g} h(z):=e^{\frac{g(z)}{\lambda}} \int_{0}^{z} h^{\prime}(\zeta) e^{-\frac{g(\zeta)}{\lambda}} d \zeta, z \in \mathbb{C}$, is continuous.

(ii) $T: X_{0} \rightarrow X_{0}, T h(z):=e^{\frac{g(z)}{\lambda}} \int_{0}^{z} h(\zeta) g^{\prime}(\zeta) e^{-\frac{g(\zeta)}{\lambda}} d \zeta, z \in \mathbb{C}$, is continuous.

Proof. This is a direct consequence of the identity

$$
e^{\frac{g(z)}{\lambda}} \int_{0}^{z} h^{\prime}(\zeta) e^{-\frac{g(\zeta)}{\lambda}} d \zeta=h(z)+(1 / \lambda) e^{\frac{g(z)}{\lambda}} \int_{0}^{z} h(\zeta) g^{\prime}(\zeta) e^{-\frac{g(\zeta)}{\lambda}} d \zeta,
$$

valid for $h \in H(\mathbb{C}), h(0)=0$, that can be seen integrating by parts. 


\section{Spectra of Volterra operators on $H_{v}^{\infty}(\mathbb{C})$}

We concentrate our attention in the Volterra operator acting on the Banach space $H_{v}^{\infty}(\mathbb{C})$, with $v(r)=\exp \left(-\alpha r^{p}\right)$, where $\alpha, p>0$. According to [13, Corollaries 3.12 and 3.13], we have the following result.

Proposition 2.1 Assume that $v(r)=\exp \left(-\alpha r^{p}\right), \alpha>0, p>0$.

(i) $V_{g}: H_{v}^{\infty}(\mathbb{C}) \rightarrow H_{v}^{\infty}(\mathbb{C})$ is continuous if and only if $g$ is a polynomial of degree less than or equal to the integer part of $p$.

(ii) $V_{g}: H_{v}^{\infty}(\mathbb{C}) \rightarrow H_{v}^{\infty}(\mathbb{C})$ is compact if and only if $g$ is a polynomial of degree less than or equal to the integer part of $p-1$.

Lemma 2.2 Let $v$ be a weight such that $v(r) e^{\alpha r^{n}}$ is non-increasing on $\left[r_{0}, \infty[\right.$ for some $r_{0}>0, \alpha>0$ and $n \in \mathbb{N}$. The operator $T: H_{v}^{\infty}(\mathbb{C}) \rightarrow H_{v}^{\infty}(\mathbb{C})$ defined by

$$
T_{\gamma} h(z):=e^{\gamma z^{n}} \int_{0}^{z} \zeta^{n-1} h(\zeta) e^{-\gamma \zeta^{n}} d \zeta, \quad z \in \mathbb{C}
$$

is continuous if $|\gamma|<\alpha$.

Proof. Changing the value of $v(r)$ on a compact interval, we may assume that $v(r) e^{\alpha r^{n}}$ is non-increasing on $[0, \infty[$. Fix $z \in \mathbb{C}, z \neq 0$. For each $0 \leq t \leq 1$ we have

$$
v(|z|) \leq v(t|z|) e^{\alpha|z|^{n}\left(t^{n}-1\right)} .
$$

Therefore we can estimate

$$
\begin{gathered}
v(|z|)\left|T_{\gamma} h(z)\right|=v(|z|)\left|\int_{0}^{1} z^{n} t^{n-1} h(t z) e^{\gamma z^{n}\left(1-t^{n}\right)} d t\right| \leq \\
\leq|z|^{n} \int_{0}^{1} t^{n-1}|h(t z)| v(t z) e^{|\gamma||z|^{n}\left(1-t^{n}\right)} e^{\alpha|z|^{n}\left(t^{n}-1\right)} d t \leq \\
\leq\|h\|_{v}|z|^{n} \int_{0}^{1} t^{n-1} e^{(\alpha-|\gamma|)|z|^{n}\left(t^{n}-1\right)} d t= \\
(1 / n)\|h\|_{v}(\alpha-|\gamma|)^{-1}\left(1-e^{-(\alpha-|\gamma|)|z|^{n}}\right) \leq(1 / n)(\alpha-|\gamma|)^{-1}\|h\|_{v},
\end{gathered}
$$

since $\alpha-|\gamma|>0$.

Theorem 2.3 Assume that $v(r)=\exp \left(-\alpha r^{p}\right), \alpha>0, p>0$. Let $g$ be a polynomial of degree $n$ less than or equal to the integer part of $p$ with $g(0)=0$.

(i) If the degree $n$ of $g$ satisfies $n<p$, then $\sigma\left(V_{g}, H_{v}^{\infty}(\mathbb{C})\right)=\{0\}$.

(ii) If $p=n \in \mathbb{N}$ and $g(z)=\beta z^{n}+k(z), k$ a polynomial of degree strictly less than $n$, then $\sigma\left(V_{g}, H_{v}^{\infty}(\mathbb{C})\right)=\{\lambda \in \mathbb{C}|| \lambda|\leq| \beta \mid / \alpha\}$.

Moreover, we have $\sigma\left(V_{g}, H_{v}^{\infty}(\mathbb{C})\right)=\{0\} \cup \overline{\left\{\lambda \in \mathbb{C} \backslash\{0\} \mid e^{\frac{g}{\lambda}} \notin H_{v}^{\infty}(\mathbb{C})\right\}}$. 
Proof. (i) If $n$ is less than or equal to the integer part of $p-1$, then $V_{g}: H_{v}^{\infty}(\mathbb{C}) \rightarrow$ $H_{v}^{\infty}(\mathbb{C})$ is compact. This implies that $\sigma\left(V_{g}, H_{v}^{\infty}(\mathbb{C})\right)=\{0\}$, since $V_{g}$ has no eigenvalues by Proposition 1.1.

Assume now that $p-1<n<p$. For each $\lambda \neq 0, e^{\frac{g}{\lambda}} \in H_{v}^{\infty}(\mathbb{C})$ as it is easy to check.

Suppose first that $g(z)=\beta z^{n}$ for some $\beta \neq 0$. For $\lambda \neq 0$, take $\alpha>|\beta| /|\lambda|$. Clearly $v(r) e^{\alpha r^{n}}$ is non-increasing on $\left[r_{0}, \infty\left[\right.\right.$ for some $r_{0}>0$. We can apply Lemma 2.2 to conclude that

$$
T_{\beta / \lambda} h(z):=e^{\frac{\beta z^{n}}{\lambda}} \int_{0}^{z} \zeta^{n-1} h(\zeta) e^{-\frac{\beta \zeta^{n}}{\lambda}} d \zeta, \quad z \in \mathbb{C},
$$

is continuous on the subspace of $H_{v}^{\infty}(\mathbb{C})$ of the functions vanishing at 0 . By Lemma 1.6, the operator

$$
S_{\lambda, g} h(z):=e^{\frac{g(z)}{\lambda}} \int_{0}^{z} h^{\prime}(\zeta) e^{-\frac{g(\zeta)}{\lambda}} d \zeta, z \in \mathbb{C},
$$

is continuous in the same Banach space. Finally, Lemma 1.5 implies $\lambda \in \rho\left(V_{g}, H_{v}^{\infty}(\mathbb{C})\right)$. This completes the proof in this case.

Suppose now that $g(z)=\beta z^{n}+k(z)$ for some $\beta \neq 0$ and some polynomial $k$ of degree strictly less than $n$. Setting $g_{1}(z):=\beta z^{n}$, we have $V_{g}=V_{g_{1}}+V_{k}$, and $V_{k}$ is a compact injective operator on $H_{v}^{\infty}(\mathbb{C})$. If $\lambda \neq 0$, we have $V_{g}-\lambda I=\left(V_{g_{1}}-\lambda I\right)+V_{k}$, and $V_{g}-\lambda I$ is bijective if and only if $V_{g_{1}}-\lambda I$ is bijective. This is a consequence e.g. of [20, Corollary 34.14] keeping in mind that both $V_{g}-\lambda I$ and $V_{g_{1}}-\lambda I$ are injective by Proposition 1.1. Therefore, we conclude $\sigma\left(V_{g}, H_{v}^{\infty}(\mathbb{C})\right)=\sigma\left(V_{g_{1}}, H_{v}^{\infty}(\mathbb{C})\right)=\{0\}$.

(ii) We suppose now that $v(r)=\exp \left(-\alpha r^{n}\right), \alpha>0$, and that $g$ is a polynomial of degree exactly $n$.

Again we consider first the case $g(z)=\beta z^{n}$. For $\lambda \in \mathbb{C} \backslash\{0\}$, we have $e^{\frac{g}{\lambda}} \in H_{v}^{\infty}(\mathbb{C})$ if and only if $|\beta| /|\lambda| \leq \alpha$. Therefore, we can apply Lemma 1.4 to conclude that $\{\lambda|| \lambda \mid \leq$ $|\beta| / \alpha\} \subset \sigma\left(V_{g}, H_{v}^{\infty}(\mathbb{C})\right)$. Now take $\lambda \in \mathbb{C}$ with $|\lambda|>|\beta| / \alpha$. Since $v(r) \exp \left(\alpha r^{n}\right)=1$, we apply Lemma 2.2 and Lemma 1.6 to get that the operator

$$
S_{\lambda, g} h(z):=e^{\frac{g(z)}{\lambda}} \int_{0}^{z} h^{\prime}(\zeta) e^{-\frac{g(\zeta)}{\lambda}} d \zeta, z \in \mathbb{C},
$$

is continuous on the subspace of $H_{v}^{\infty}(\mathbb{C})$ of the functions vanishing at 0 . Consequently $\lambda \in \rho\left(V_{g}, H_{v}^{\infty}(\mathbb{C})\right)$ by Lemma 1.5 , which yields $\sigma\left(V_{g}, H_{v}^{\infty}(\mathbb{C})\right)=\{\lambda \in \mathbb{C}|| \lambda|\leq| \beta \mid / \alpha\}$ in the present case.

In the general case $g(z)=\beta z^{n}+k(z), \beta \neq 0$ and some polynomial $k$ of degree strictly less than $n$, we proceed as in the proof of part (i) to conclude $\sigma\left(V_{g}, H_{v}^{\infty}(\mathbb{C})\right)=$ $\sigma\left(V_{g_{1}}, H_{v}^{\infty}(\mathbb{C})\right), g_{1}(z)=\beta z^{n}$.

Given a weight $v$, one defines the following closed subspace of $\left.H_{v}^{\infty}(\mathbb{C})\right)$ :

$$
H_{v}^{0}(\mathbb{C}):=\left\{f \in H(\mathbb{C})\left|\lim _{|z| \rightarrow \infty} v(|z|)\right| f(z) \mid=0\right\} .
$$

The polynomials are contained and dense in $H_{v}^{0}(\mathbb{C})$. By $[12$, Ex 2.2], the bidual of $H_{v}^{0}(\mathbb{C})$ is isometrically isomorphic to $H_{v}^{\infty}(\mathbb{C})$. By [13, Corollaries 3.8 and 3.12] $V_{g}$ is bounded on $H_{v}^{0}(\mathbb{C})$ if and only if it is bounded on $H_{v}^{\infty}(\mathbb{C})$ when $v(r)=\exp \left(-\alpha r^{p}\right), \alpha, p>0$. Now, proceeding as in the proof of [5, Lemma 1] or [6, Lemma 2.1] one can show, for $v(r)=\exp \left(-\alpha r^{p}\right)$, that if $V_{g}$ is bounded on $H_{v}^{\infty}(\mathbb{C})$, then it coincides with the bi-transpose $\left(V_{g}\right)^{\prime \prime}$ of $V_{g}: H_{v}^{0}(\mathbb{C}) \rightarrow H_{v}^{0}(\mathbb{C})$. Accordingly, $\sigma\left(V_{g}, H_{v}^{0}(\mathbb{C})\right)=\sigma\left(V_{g}, H_{v}^{\infty}(\mathbb{C})\right)$. 


\section{Spectra of Volterra operators on Hörmander algebras}

A function $p: \mathbb{C} \rightarrow[0, \infty[$ is called a growth condition if it is continuous, subharmonic, radial, increases with $|z|$ and satisfies:

$(\alpha) \log \left(1+|z|^{2}\right)=o(p(|z|))$ as $|z| \rightarrow \infty$,

$(\beta) p(2|z|)=O(p(|z|))$ as $|z| \rightarrow \infty$.

Given a growth condition $p$, we define the following weighted spaces of entire functions (see e.g. [8] and [9]):

$$
A_{p}:=\left\{f \in H(\mathbb{C}) \mid \text { there is } A>0: \sup _{z \in \mathbb{C}}|f(z)| \exp (-A p(z))<\infty\right\}
$$

endowed with the inductive limit topology, for which it is a (DFN)-algebra (cf. [23]). We also define

$$
A_{p}^{0}:=\left\{f \in H(\mathbb{C}) \mid \text { for all } \varepsilon>0: \sup _{z \in \mathbb{C}}|f(z)| \exp (-\varepsilon p(z))<\infty\right\},
$$

endowed with the projective limit topology, for which it is a nuclear Fréchet algebra (cf. $[24])$.

Clearly $A_{p}^{0} \subset A_{p}$. Condition $(\alpha)$ implies that the polynomials are dense in $A_{p}$ and in $A_{p}^{0}$. Condition $(\beta)$ implies that both spaces are stable under differentiation. By the closed graph theorem, the differentiation operator $D$ is continuous on $A_{p}$ and on $A_{p}^{0}$. It was observed in [7, Lemma 4.1] that the integration operator $J$ is continuous on both spaces.

Weighted algebras of entire functions of this type, usually known as Hörmander algebras, have been considered since the work of Berenstein and Taylor [9] by many authors; see e.g. [8] and the references therein. Braun, Meise and Taylor studied in [14], [23] and [24] the structure of (complemented) ideals in these algebras. As an example, when $p(z)=|z|^{a}$, then $A_{p}$ consists of all entire functions of order $a$ and finite type or order less than $a$, and $A_{p}^{0}$ is the space of all entire functions of order at most $a$ and type 0 . For $a=1, A_{p}$ is the space of all entire functions of exponential type, and $A_{p}^{0}$ is the space of entire functions of infraexponential type.

The following Lemma, that is a consequence of condition $(\beta)$ for the growth condition $p$, is well known.

Lemma 3.1 If $p: \mathbb{C} \rightarrow[0, \infty[$ is a growth condition, then there are $M>0$ and $s>0$ such that $p(r) \leq M r^{s}+M$ for each $r \in[0, \infty[$.

Proposition 3.2 Let $g$ be an entire function.

(i) $V_{g}: A_{p} \rightarrow A_{p}$ is continuous if and only if $g \in A_{p}$.

(ii) $V_{g}: A_{p}^{0} \rightarrow A_{p}^{0}$ is continuous if and only if $g \in A_{p}^{0}$.

Proof. We show (i), the proof of (ii) being similar. The identities $D \circ V_{g}=M_{g^{\prime}}, V_{g}=$ $J \circ M_{g^{\prime}}$ and the continuity of $D$ and $J$ on $A_{p}$ imply that $V_{g}: A_{p} \rightarrow A_{p}$ is continuous if and only the operator of multiplication $M_{g^{\prime}}: A_{p} \rightarrow A_{p}$ is continuous. Since $A_{p}$ is an algebra containing the constants, this holds if and only if $g^{\prime} \in A_{p}$. We can apply again that $D$ and $J$ are continuous on $A_{p}$ to conclude that this statement is equivalent to $g \in A_{p}$. 
The spectrum of the integration operator $J$ on $A_{p}$ and $A_{p}^{0}$ was investigated in [7, Proposition 4.7]. This result, that corresponds to $V_{g}$ for $g(z)=z$, is extended in this section.

Lemma 3.3 Let $p: \mathbb{C} \rightarrow[0, \infty[$ be a growth condition and let $h$ be an entire function.

(i) The function $e^{h}$ belongs to $A_{p}$ if and only if $M(h, r)=O(p(r))$ as $r \rightarrow \infty$. If this is the case, then $h$ is a polynomial.

(ii) The function $e^{h}$ belongs to $A_{p}^{0}$ if and only if $M(h, r)=o(p(r))$ as $r \rightarrow \infty$. If this is the case, then $h$ is a polynomial.

Proof. (i) If $M(h, r)=O(p(r))$ as $r \rightarrow \infty$, then $e^{h}$ clearly belongs to $A_{p}$. To prove the converse, denote by $A(h, r)$ the maximum of the real part of $h$ in the circle $|z| \leq r$ for $r>0$. As $e^{h} \in A_{p}$, there is $C>0$ such that $A(h, r) \leq C p(r)+C$ for each $r>0$. We apply Carathéodory inequality for the circle $|z| \leq 2 r$ (c.f. [21, Theorem 6.8]) and property $(\beta)$ of $p$ to get

$$
M(h, r) \leq 2(A(h, 2 r)-\operatorname{Re} h(0))+|h(0)| \leq 2 C p(2 r)+2(C+|h(0)|) \leq C^{\prime} p(r)+C^{\prime} .
$$

Now Lemma 3.1 and a standard argument imply that $h$ is a polynomial.

(ii) By [24, Lemma 2.3], $e^{h} \in A_{p}^{0}$ if and only if there is a growth condition $q$ such that $e^{h} \in A_{q}$ and $q(r)=o(p(r)), r \rightarrow \infty$. By part (i), $M(h, r)=O(q(r))=o(p(r)), r \rightarrow \infty$. The reverse implication is trivial.

Theorem 3.4 Let $p: \mathbb{C} \rightarrow\left[0, \infty\left[\right.\right.$ be a growth condition and let $g \in A_{p}$ be non-constant.

(i) If $M(g, r)=O(p(r)), r \rightarrow \infty$, is not satisfied (which happens in particular when $p(r)=o(r), r \rightarrow \infty)$, then $\sigma\left(V_{g}, A_{p}\right)=\mathbb{C}$.

(ii) If $M(g, r)=O(p(r)), r \rightarrow \infty$, then $\sigma\left(V_{g}, A_{p}\right)=\{0\}$. In this case $g$ is a polynomial and $r=O(p(r)), r \rightarrow \infty$.

Moreover, in both cases we have $\sigma\left(V_{g}, A_{p}\right)=\{0\} \cup \overline{\left\{\lambda \in \mathbb{C} \backslash\{0\} \mid e^{\frac{g}{\lambda}} \notin A_{p}\right\}}$.

Proof. First observe that $M(g / \lambda, r)=(1 /|\lambda|) M(g, r)$ for each $\lambda \in \mathbb{C} \backslash\{0\}$ and $r>0$. Therefore, it follows from Lemma 3.3 (i) that $e^{\frac{g}{\lambda}} \in A_{p}$ for some (all) $\lambda \neq 0$, if and only if $e^{g} \in A_{p}$, that is equivalent to $M(g, r)=O(p(r))$ as $r \rightarrow \infty$.

(i) If $M(g, r)=O(p(r))$ as $r \rightarrow \infty$ is not satisfied, then $e^{\frac{g}{\lambda}} \notin A_{p}$ for each $\lambda \neq 0$ by our remarks above. We can apply Lemma 1.4 to conclude $\sigma\left(V_{g}, A_{p}\right)=\mathbb{C}$.

Observe that in case $p(r)=o(r), r \rightarrow \infty$, then $M(g, r)=O(p(r))$ as $r \rightarrow \infty$ is not satisfied, since otherwise we would have $M(g, r)=O(p(r))=o(r)$ as $r \rightarrow \infty$, that implies that $g$ is constant; a contradiction.

(ii) If $M(g, r)=O(p(r)), r \rightarrow \infty$, then $e^{\frac{g}{\lambda}} \in A_{p}$ for each $\lambda \neq 0$. Given $\lambda \in \mathbb{C} \backslash\{0\}$, setting $G=e^{\frac{g}{\lambda}}, 1 / G=e^{-\frac{g}{\lambda}}$, the operator $S_{\lambda, g}$ of Lemma 1.5 (iii) satisfies $S_{\lambda, g}=M_{G} \circ J \circ$ $M_{1 / G} \circ D$. These four operators are continuous on the algebra $A_{p}$. Therefore $\lambda \in \rho\left(V_{g}, A_{p}\right)$, and $\sigma\left(V_{g}, A_{p}\right)=\{0\}$.

In this case, since $g$ must be a non constant polynomial, the assumption in (ii) implies $r=O(p(r)), r \rightarrow \infty$. 
Theorem 3.5 Let $p: \mathbb{C} \rightarrow\left[0, \infty\left[\right.\right.$ be a growth condition and let $g \in A_{p}^{0}$ be non-constant.

(i) If $M(g, r)=o(p(r)), r \rightarrow \infty$, is not satisfied (which happens in case $p(r)=O(r), r \rightarrow$ $\infty)$, then $\sigma\left(V_{g}, A_{p}^{0}\right)=\mathbb{C}$.

(ii) If $M(g, r)=o(p(r)), r \rightarrow \infty$, then $\sigma\left(V_{g}, A_{p}^{0}\right)=\{0\}$. In this case $g$ is a polynomial and $r=o(p(r)), r \rightarrow \infty$.

Moreover, in both cases, we have $\sigma\left(V_{g}, A_{p}^{0}\right)=\{0\} \cup \overline{\left\{\lambda \in \mathbb{C} \backslash\{0\} \mid e^{\frac{g}{\lambda}} \notin A_{p}^{0}\right\}}$.

Proof. Since $M(g / \lambda, r)=(1 /|\lambda|) M(g, r)$ for each $\lambda \in \mathbb{C} \backslash\{0\}$ and $r>0$, Lemma 3.3 (ii) implies that $e^{\frac{g}{\lambda}} \in A_{p}^{0}$ for some (or all) $\lambda \neq 0$ if and only if $e^{g} \in A_{p}^{0}$.

(i) If $M(g, r)=o(p(r))$ as $r \rightarrow \infty$ is not satisfied, then $e^{\frac{g}{\lambda}} \notin A_{p}^{0}$ for each $\lambda \neq 0$ by our comments above. By Lemma 1.4 we have $\sigma\left(V_{g}, A_{p}\right)=\mathbb{C}$.

Observe that in case $p(r)=O(r), r \rightarrow \infty$, then $M(g, r)=o(p(r))$ as $r \rightarrow \infty$ does not hold. Otherwise, $M(g, r)=o(p(r))=O(r)$ as $r \rightarrow \infty$, which implies that $g$ is constant; a contradiction.

(ii) If $M(g, r)=o(p(r)), r \rightarrow \infty$, then $e^{\frac{g}{\lambda}} \in A_{p}^{0}$ for each $\lambda \neq 0$ by our comments above. Given $\lambda \in \mathbb{C} \backslash\{0\}$, setting $G=e^{\frac{g}{\lambda}}$, the operator $S_{\lambda, g}$ of Lemma 1.5 (iii) satisfies $S_{\lambda, g}=M_{G} \circ J \circ M_{1 / G} \circ D$ and is continuous on the algebra $A_{p}^{0}$. Therefore $\lambda \in \rho\left(V_{g}, A_{p}^{0}\right)$.

Since $g$ must be a non constant polynomial, the assumption in (ii) yields $r=o(p(r))$ as $r \rightarrow \infty$.

Acknowledgement. This research was partially supported by MINECO Project MTM2013-43540-P and by GV Project Prometeo II/2013/013.

\section{References}

[1] A. Aleman, O. Constantin, Spectra of integration operators on weighted Bergman spaces, J. Anal. Math. 109 (2009), 199-231.

[2] A. Aleman, J.A. Peláez, Spectra of integration operators and weighted square functions, Indiana Univ. Math. J. 61 (2012), 1-19.

[3] A. Aleman, A.G. Siskakis, An integral operator on $H^{p}$, Complex Var. Theory Appl. 28 (1995), 149-158.

[4] A. Aleman, A.G. Siskakis, Integration operators on Bergman spaces, Indiana Univ. Math. J. 46 (1997), 337-356.

[5] M. Basallote, M.D. Contreras, C. Hernández-Mancera, M.J. Martín, P.J. Paúl, Volterra operators and semigroups in weighted Banach spaces of analytic functions, Collect. Math. 65 (2014), 233-249.

[6] M.J. Beltrán, J. Bonet, C. Fernández, Classical operators on weighted Banach spaces of entire functions, Proc. Amer. Math. Soc. 141 (2013), 4293-4303.

[7] M.J. Beltrán, J. Bonet, C. Fernández, Classical operators on the Hörmander algebras, Discr. Cont. Dynam. Syst. 35 (2015), 637-652.

[8] C. A. Berenstein and R. Gay. Complex analysis and special topics in harmonic analysis. Springer-Verlag, New York, 1995. 
[9] C. Berenstein and B. A. Taylor, A new look at interpolation theory for entire functions of one variable, Adv. in Math. 33 (1979), 109-143.

[10] K.D. Bierstedt, J. Bonet, A. Galbis, Weighted spaces of holomorphic functions on balanced domains, Michigan Math. J. 40 (1993), no. 2, 271-297.

[11] K.D. Bierstedt, J. Bonet, J. Taskinen, Associated weights and spaces of holomorphic functions, Studia Math. 127 (1998), 137-168.

[12] K.D. Bierstedt, W. H. Summers, Biduals of weighted Banach spaces of analytic functions, J. Austral. Math. Soc. Ser. A 54 (1993), no. 1, 70-79.

[13] J. Bonet, J. Taskinen, A note about Volterra operators on weighted Banach spaces of entire functions, Math. Nachr. (to appear).

[14] R. W. Braun, Weighted algebras of entire functions in which each closed ideal admits two algebraic generators, Michigan Math. J. 34 (1987), 441-450.

[15] O. Constantin, A Volterra-type integration operator on Fock spaces, Proc. Amer. Math. Soc. 140 (2012), 4247-4257.

[16] O. Constantin, J.A. Peláez, Integral operators, embedding theorems and a LittlewoodPaley formula on weighted Fock spaces, arXiv:1304.7501v1.

[17] O. Constantin, J.A. Peláez, Boundedness of the Bergman projection on $L^{p}$ spaces with exponential weights, arXiv: 1309.6071v2.

[18] M. Contreras, A.G. Hernández-Díaz, Weighted composition operators in weighted Banach spacs of analytic functions, J. Austral. Math. Soc. (Series A) 69 (2000), 41-60.

[19] A. Harutyunyan, W. Lusky, On the boundedness of the differentiation operator between weighted spaces of holomorphic functions, Studia Math. 184 (2008), 233-247.

[20] G.J.O. Jameson, Topology and Normed Spaces, Chapman Hall, London, 1974.

[21] B. Ya. Levin, Distribution of zeros of entire functions. Translated from the Russian by R. P. Boas, J. M. Danskin, F. M. Goodspeed, J. Korevaar, A. L. Shields and H. P. Thielman. Revised edition. Translations of Mathematical Monographs, 5. American Mathematical Society, Providence, R.I., 1980.

[22] W. Lusky, On the isomorphism classes of weighted spaces of harmonic and holomophic functions, Studia Math. 75 (2006) 19-45.

[23] R. Meise, Sequence space representations for (DFN)-algebras of entire functions modulo closed ideals, J. Reine Angew. Math. 363 (1985), 59-95.

[24] R. Meise and B. A. Taylor, Sequence space representations for (FN)-algebras of entire functions modulo closed ideals, Studia Math. 85 (1987), 203-227.

[25] R. Meise and D. Vogt. Introduction to functional analysis, volume 2 of Oxford Graduate Texts in Mathematics. The Clarendon Press Oxford University Press, New York, 1997.

[26] J. Pau, J.A. Peláez, Embedding theorems and integration operators on Bergman spaces with rapidly decreasing weights, J. Funct. Anal. 259 (2010), 2727-2756. 
[27] J.A. Peláez, J. Rättyä, Weighted Bergman Spaces induced by rapidly decreasing weights, Memoirs Amer. Math. Soc. 227, 2014.

[28] Ch. Pommerenke, Schlichte Funktionen un analytische Functionen von beschrükter mittlerer Oszilation, Comment. Math. Helv. 52 (1977), 591-602.

[29] J.H. Shapiro, Composition Operators and Classical Function Theorey, Springer, New York, 1993.

\section{Author's address:}

José Bonet: Instituto Universitario de Matemática Pura y Aplicada IUMPA, Universitat Politècnica de València, E-46071 Valencia, Spain

email: jbonet@mat.upv.es 